\title{
Association of AFF3 Gene Polymorphism rs10865035 with Rheumatoid Arthritis: A Population-Based Case-Control Study on a Pakistani Cohort
}

\author{
Yasir Ali $\mathbb{D}^{1},{ }^{1}$ Suleman Khan, ${ }^{2}$ Yangchao Chen, ${ }^{3}$ Nadia Farooqi, ${ }^{1}$ Zia-Ul Islam, \\ Mehran Akhtar $\left(\mathbb{D},{ }^{1,4}\right.$ Aamir, ${ }^{1}$ Aisha Aman, ${ }^{1}$ Aftab Ali Shah, ${ }^{5}$ Muhsin Jamal, ${ }^{6}$ and Fazal Jalil $\left(\mathbb{1}{ }^{1}\right.$ \\ ${ }^{1}$ Department of Biotechnology, Abdul Wali Khan University Mardan, Mardan, Pakistan \\ ${ }^{2}$ Lady Ready Hospital, MTI Peshawar, Peshawar, Pakistan \\ ${ }^{3}$ School of Biomedical Sciences, Chinese University of Hong Kong, Hong Kong, Pakistan \\ ${ }^{4}$ Department of Biotechnology, COMSATS University Islamabad, Abbottabad Campus, Abbottabad, Pakistan \\ ${ }^{5}$ Department of Biotechnology, University of Malakand, Chakdara, Pakistan \\ ${ }^{6}$ Department of Microbiology, Abdul Wali Khan University Mardan, Mardan, Pakistan
}

Correspondence should be addressed to Fazal Jalil; fazaljalil@awkum.edu.pk

Received 27 January 2021; Revised 9 April 2021; Accepted 29 April 2021; Published 17 May 2021

Academic Editor: John Charles Rotondo

Copyright () 2021 Yasir Ali et al. This is an open access article distributed under the Creative Commons Attribution License, which permits unrestricted use, distribution, and reproduction in any medium, provided the original work is properly cited.

\begin{abstract}
Rheumatoid arthritis (RA) is one of the complex diseases with the involvement of the genetic as well as environmental factors in its onset and severity. Different genome-wide association and candidate gene studies have shown the role of several genetic variants in multiple loci/genes with ethnical and geographical variations. This study was designed to detect the association of a singlenucleotide polymorphism (SNP) rs10865035 in the AFF3 gene with the genetic background of rheumatoid arthritis (RA) in the Pakistani cohort. A total of 703 individuals, including 409 RA patients and 294 healthy controls, were genotyped using TaqMan assay and Tri primer ARMS-PCR (amplification-refractory mutation system-polymerase chain reaction) methods. The association of rs10865035 with the RA was statistically determined using different models. Interestingly, besides the homozygous recessive model $(\mathrm{G} / \mathrm{G}$ vs. $\mathrm{A} / \mathrm{G}+\mathrm{A} / \mathrm{A})(\mathrm{OR}=1.693(1.06-2.648) ; P=0.025)$, all other models, which included the codominant $\left(\chi^{2}=5.169\right.$; $P=0.075)$, homozygous dominant (A/A vs. $\mathrm{G} / \mathrm{G}+\mathrm{A} / \mathrm{G})(\mathrm{OR}=0.867(0.636-1.187) ; P=0.41)$, heterozygous $(\mathrm{A} / \mathrm{G}$ vs. A/A + GG) $(\mathrm{OR}=0.491(0.667-1.215) ; P=0.49)$, and additive model $(\mathrm{OR}=0.826(0.665-1.027) ; P=0.08)$ showed insignificant distribution of the genotypes among the cases and controls. These findings suggest that the AFF3 gene (rs10865035) has no significant role in the onset of RA in the Pakistani population.
\end{abstract}

\section{Introduction}

Rheumatoid arthritis (RA) is a systemic, chronic, and progressive inflammatory disease. This disease mainly affects multiple joints as well as exhibits extra-articular manifestations such as rheumatoid nodules, systemic comorbidities, and pulmonary involvement of vacuities. The therapeutic revolution in the past few decades that includes the advancement of the new therapeutic options, the development of new criteria for classification, the introduction of early therapy, and the application of new effective strategies has transformed the articular and systemic outcomes in RA $[1,2]$. The modern genetic technologies combined with the large and well-characterized clinical cohorts have advanced our understanding of the genetics of this disease. Genomewide association studies (GWAS) have reported more than a hundred loci associated with the RA risk [3-5]. The presence of genetic polymorphisms in HLA (human leukocyte antigen) and non-HLA genes contributes about $60 \%$ to the pathogenesis of RA. Out of HLA genes, the HLA-DRB1 and associated polymorphisms are crucial in genetic susceptibility of RA, whereas other important non-HLA genes are 
TABLE 1: Characteristic of rheumatoid arthritis patients and controls.

\begin{tabular}{lcc}
\hline Characteristics & Cases $(n=409)$ & Controls $(n=294)$ \\
\hline Group A* & 159 & 44 \\
Group B & 250 & 250 \\
Mean age, years \pm SD of group A* & $39.1 \pm 13.0$ & $41.2 \pm 12.0$ \\
Mean age, years \pm SD group B & $43.5 \pm 14.5$ & $42 \pm 12.6$ \\
Disease duration in years, mean \pm SD & $4.1 \pm 3.7$ & NIL \\
Seropositive antibody, mean \pm SD & $100 \%($ RF positive) & NIL \\
ESR, mean \pm SD & $40.60( \pm 15.8)$ & NIL \\
\hline
\end{tabular}

$*$ Data obtained from our previous study [7].

PTPN22, IL23R, TRAF1, CTLA4, IRF5, AFF3, STAT4, CCR6, and PADI4, etc. [6-9].

The AFF3 (lymphoid nuclear protein related to the AF4 gene) is located at $2 \mathrm{q} 11.2$ position and encodes a protein of 1227 amino acids. This gene is highly expressed in the lymphoid tissue and has been suggested to be involved in its development, while a lower level of expression has been reported in other tissues like the brain and lungs [1, 10-12]. This gene forms a nuclear factor that binds to DNA through its transcriptional activation domain. This property of the AFF3 gene has made it a strong candidate for autoimmunity in the human cell [13-15]. A large number of studies, including the genome-wide association studies and their metaanalysis, conducted in different populations, have investigated the association of AFF3 rs10865035, rs1160542, rs17023158, and rs1437377 variants in the pathogenesis of RA $[13,16-20]$.

The AFF3 gene is not only important in the susceptibility to RA but also is a good candidate in the assessment of the therapeutic response in individuals [12]. The SNPs in any population are helpful in understanding its genetic diversity and to design a population-specific therapy for RA. Previously, European RA risk loci including AFF3 rs10865035 were replicated in a small sample set of Pakistani individuals [7]. However, in this study, the association of AFF3 rs10865035 with RA was investigated in another sample set of Pakistani origin and was merged with our previous data for statistical significance.

\section{Methodology}

2.1. Study Subjects. A cohort of 703 individuals of Pakistani origin (409 RA cases and 294 healthy controls) were included in this study after getting their informed consent. The study cohort was divided into group $\mathrm{A}(n=203)$ and group $\mathrm{B}$ $(n=500)$. The dataset of group A was taken from our previous study [7]. The details of group B are given in Table 1 . The cases were clinically diagnosed by certified rheumatologists following American College of Rheumatology (ACR) criteria [1], and the data including their clinical and demographic information were recorded on a specially designed questionnaire. A comparison of the RA cases and controls showed that the level of inflammatory markers like rheumatoid factor (RF) and erythrocyte sedimentation rate (ESR) was significantly higher in cases. The participants having no immunological disease/symptoms were counted as controls in this study. The study protocol was approved by the Ethical Review Board of LRH and AWKUM (Abdul Wali khan University Mardan).

2.2. DNA Extraction and Genotyping. Whole blood samples were collected and processed for group B $(n=500)$ as previously done by Jalil et al., 2013, for group A $(n=203)$. The genomic DNA was extracted using an organic phenolchloroform method [21]. The genotyping of the AFF3 gene variant rs10865035 was carried out using TaqMan genotype SNP assay (Applied Biosystems) and ARMS-PCR techniques. The individuals in group $\mathrm{A}(n=203)$ were analyzed through TaqMan assay where the PCR amplification was performed in 384-well plates on dual block GeneAmp PCR system 9700 (Applied Biosystems), following the manufacture's protocol [7]. The remaining 500 samples were analyzed through ARMS-PCR using a set of three primers, including two forward and a common reverse primer (F1 (for A allele): TTTAAAACCTCTATCTGGGGAAAAA, F2 (for $\mathrm{G}$ allele): TAAAACCTCTATGGGGAAAAG and R: CCCCTCTAATAGTCAATCAATCAAAATA).

The PCR amplification was performed in 96-well plates on a thermocycler (T100, Bio-Rad). The amplification conditions were set as the initial denaturation at $94^{\circ} \mathrm{C}$ for 5 minutes, followed by 35 cycles of denaturation at $94^{\circ} \mathrm{C}$ for 30 seconds, annealing for 30 seconds at $58^{\circ} \mathrm{C}$, and extension at $72^{\circ} \mathrm{C}$ for 1 minute. The final elongation was performed at $72^{\circ} \mathrm{C}$ for 5 minutes. The PCR products were resolved in $2 \%$ agarose gel, and the genotype calls for each subject were recorded using the visual inspection method of the gel.

2.3. Statistical Analysis. The association of AFF3 rs 10865035 with RA was tested through different statistical models such as codominant, homozygous dominant, homozygous recessive, heterozygous, and additive. The data were further checked for deviation from the Hardy-Weinberg Equilibrium (HWE), and the error in genotyping was measured by repeating $10 \%$ of the samples. The association of AFF3 rs10865035 with RA was measured by calculating the odds ratio $(\mathrm{OR})$ at $95 \%$ confidence interval $(\mathrm{CI})$ using chi-square $\left(\chi^{2}\right)$ and Fisher's exact test. The $P$ value $<0.05$ was considered statistically significant.

\section{Results}

The data of the current study $(n=500)$ were combined with our previous findings $(n=203)$ to make the sample size more 
TABLE 2: Statistical analysis of the AFF3 polymorphism (rs340630) in RA cases and controls.

\begin{tabular}{|c|c|c|c|c|c|c|}
\hline Statistical models & Genotypes & Cases $(n=409)$ & Controls $(n=294)$ & OR 95\% CI & $\chi^{2}$ value & $P$ value \\
\hline \multirow{3}{*}{ Codominant } & $\mathrm{A} / \mathrm{A}$ & $133(32.52 \%)$ & $105(35.72 \%)$ & & \multirow{3}{*}{5.169} & \multirow{3}{*}{0.07} \\
\hline & $\mathrm{A} / \mathrm{G}$ & $210(51.35 \%)$ & $159(54.09 \%)$ & - & & \\
\hline & $\mathrm{G} / \mathrm{G}$ & $66(16.14 \%)$ & $301(0.21 \%)$ & & & \\
\hline \multirow{2}{*}{ Homozygous dominant } & $\mathrm{A} / \mathrm{A}$ & $133(32.5 \%)$ & $105(35.7 \%)$ & 0.867 & \multirow{2}{*}{-} & \multirow[t]{2}{*}{0.41} \\
\hline & $\mathrm{A} / \mathrm{G}+\mathrm{G} / \mathrm{G}$ & $276(67.5 \%)$ & $189(64.3 \%)$ & $(0.636-1.187)$ & & \\
\hline \multirow{2}{*}{ Homozygous recessive } & $\mathrm{G} / \mathrm{G}$ & $66(16.1 \%)$ & $30(10.2 \%)$ & 1.693 & \multirow{2}{*}{-} & \multirow[t]{2}{*}{0.02} \\
\hline & $\mathrm{A} / \mathrm{A}+\mathrm{A} / \mathrm{G}$ & $343(83.9 \%)$ & $264(89.8 \%)$ & $(1.06-2.648)$ & & \\
\hline \multirow{2}{*}{ Heterozygous dominant } & $\mathrm{A} / \mathrm{G}$ & $210(51.3 \%)$ & $159(54 \%)$ & 0.491 & \multirow[b]{2}{*}{-} & \multirow[t]{2}{*}{0.49} \\
\hline & $\mathrm{A} / \mathrm{A}+\mathrm{G} / \mathrm{G}$ & $199(48.7 \%)$ & $135(46 \%)$ & $(0.667-1.215)$ & & \\
\hline \multirow{2}{*}{ Additive } & $\mathrm{A}$ & $476(58.1 \%)$ & $369(62.3 \%)$ & 0.826 & \multirow{2}{*}{-} & \multirow[t]{2}{*}{0.08} \\
\hline & G & $342(41.9 \%)$ & $219(37.3 \%)$ & $(0.665-1.027)$ & & \\
\hline
\end{tabular}

effective. In the codominant model, the frequency of the genotypes was found almost similar in both the cases and controls (cases: A/A 133 (32.52\%), A/G 210 (51.35\%), and G/ G 66 (16.14\%); controls: A/A 105 (35.72\%), A/G 159 (54.09\%), and G/G 30 (10.21\%), $\left.\chi^{2}=5.169 ; P=0.07\right)$. Similarly, in the homozygous dominant model, no significant difference was observed in the distribution of A/A vs. G/ $\mathrm{G}+\mathrm{A} / \mathrm{G}$ genotypes in the study subjects $(\mathrm{OR}=0.867$ (0.636-1.187); $P=0.41)$. However, a significant distribution was determined in the homozygous recessive model by comparing $\mathrm{G} / \mathrm{G}$ vs. $\mathrm{A} / \mathrm{G}+\mathrm{A} / \mathrm{A}$ (OR=1.693 (1.06-2.648); $\mathrm{Pe}=0.02)$. Furthermore, an insignificant distribution of $A / G$ vs. A/A + GG genotypes was observed in the heterozygous model $(\mathrm{OR}=0.491(0.667-1.215) ; P=0.49)$ whereas in the additive model, the alleles (A vs. G) were distributed insignificantly among the cases and controls $(\mathrm{OR}=0.826$ (0.665-1.027); $P=0.08)$. The association results, $P$ values along with ORs, are given in Table 2.

\section{Discussion}

Rheumatoid arthritis (RA) is an autoimmune disease that disrupts the normal physical activities of the patients $[22,23]$. It causes inflammation in joints which results in damaging the articular cartilage along with the synovial hyperplasia that ultimately leads to consistent pain and permanent disability. In RA, the genetic risk factors (50-60\%) are triggered by various environmental factors (40\%) [23-25]. Among the genetic factors, the major histocompatibility complex (MHC) and non-MHC loci are accountable for $\sim 23 \%$ of the genetic risk which indicates that there are still undiscovered risk alleles [18]. Furthermore, the association studies have also reported $>150$ SNPs located at more than 70 gene loci $[18,26-28]$. Another gene-based association study that was conducted on European $(14,361$ cases and 43,923 controls) and Asian populations (4,873 cases and 17,642 controls) identified 221 newly RA-associated genes including 76 European-specific, 74 Asianspecific, and 71 genes which were found overlapped among both the populations [5]. Other studies that followed different study protocols have either validated the role of some of the reported genes or have determined the association of novel genes and polymorphisms in both European and Asian individuals $[1,7,17,18,29,30]$.
The aim of this study was to assess the association of the AFF3 rs10865035 with the genetic background of RA in the Pakistani population. The replication of known genetic polymorphism will enhance our understanding of the ethnogenetic heterogeneity and homogeneity of the Pakistani population with others. The understanding of replication patterns among different populations would help us in the development of a common therapy for disease management.

Previously, we determined an insignificant association $(P=0.117)$ of $A F F 3$ gene polymorphism rs10865035 in a small sample set $(n=213)$ of Pakistani origin [7]. Therefore, the current study was aimed to replicate these findings in a larger sample $(n=500)$ set of the same ethnicity. To enhance the statistical significance of this study, our previous data of 203 individuals were merged with the current data of 500 subjects. These statistical analyses showed that AFF3 gene polymorphism rs 10865035 has no association with RA in the Pakistani population (Table 2).

The AFF3 gene encodes a DNA interacting nuclear factor having a transcriptional activation domain that makes it a strong candidate gene in autoimmunity. Previously, more than 50 genetic polymorphisms have been mapped in the same gene [13]. Also, an extended study was conducted on a sample set of 6819 RA patients and 12650 healthy controls, provided a convincing proof for the association of AFF3 gene variants rs10865035 (OR: 1.12 (1.07-1.17); $P=2.8 \times 10^{-7}$ ) and rs1160542 (OR: 1.12 (1.05-1.20); $P=0.001)$ as novel susceptibility targets [13]. Using these findings, another group of researchers investigated the role of AFF3 gene polymorphisms (rs10865035 and rs1160542) with respect to anti-TNF treatment in RA (coefficient -0.14 (95\% CI -0.25 to -0.03$), P=0.01$ ) [31]. Furthermore, a GWAS meta-analysis by Stahl et al. (2010) also confirmed the association of AFF3 gene polymorphism rs10865035 (OR: $1.12(1.07-1.17) ; P=2.0 \times 10^{-6}$ ) with RA in a sample set of 41,282 individuals (12,307 RA cases and 28,975 controls) of European descent [18].

In order to explore population-specific as well as European-overlap susceptibility loci/genes, a GWAS was carried out in the Korean population. In this study, 1519 RA cases and 1476 healthy controls were genotyped for 441,398 single-nucleotide polymorphisms, and along with many other susceptibility signals, AFF3 rs10865035 was successfully associated with the RA at genome-wide significance 
level $\left(P<5 \times 10^{-08}\right)$ [19]. Another replication study, conducted by Prasad et al. [17] on 983 RA cases and 1007 controls of North Indian descent, established the association of seven genes with the RA. They selected 42 candidate genes/loci (3 Asian and 39 European) and tested 603 SNPs, which were either index SNPs or were surrogate SNPs, using Infinium Human $660 \mathrm{w}$-quad microarray methods for genotyping. Out of 12 SNPs, tested in AFF3 gene, 3 SNPs were found to be significantly associated with RA (rs17023158, OR: 1.45 (1.12-1.88), $P=0.005$; rs6706188, OR: 0.81 (0.7-0.94), $P=0.005$; rs1437377, OR: 0.77 (0.65-0.92), $P=0.003)$ [17]. However, rs10865035 was not included in their SNPs array. Similarly, a sequenom mass array-based meta-analysis conducted on European individuals (3311 RA cases and 3709 controls) investigated another SNP rs1160542 and obtained a significant association with RA (OR: 1.08 (1.01-1.16), $P=0.029$ ) [20]. In addition, the AFF3 gene has been detected to be significantly associated (OR: $\left.1.25(1.13-1.39) ; P=2.05 \times 10^{-5}\right)$ with juvenile idiopathic arthritis (JIA) as well [16]. It was further reported that, besides, its role in RA, the AFF3 gene shares the same genetic basis with the systemic lupus erythematosus (SLE). A Chinese case-control study (868 SLE patients and 975 controls) observed significant association of the AFF3 rs10865035 with SLE (OR: 1.26 (1.11-1.44); $P=4.81 \times 10^{-4}$ ) [32]. Furthermore, studies have shown that the AFF3 gene was initially linked to type 1 diabetes (T1D), and then, later, its role was determined in 16 different autoimmune diseases including RA and SLE [33,34].

In conclusion, our previous study [7] regarding the association of AFF3 rs10865035 with RA was further validated using different statistical models in a larger sample set $(n=703)$. This reaffirmation with different statistical tools suggests the nonsignificance of AFF3 rs10865035 in a Pakistani cohort. However, there is a possibility of the existence of ethnic variability and we urge the need for multiethnic large population-based study in order to understand the exact mechanism of pathogenicity as well as the evolutionary background of the genetic factors involved.

\section{Data Availability}

All the data used to support the findings of this study are cited in the text.

\section{Disclosure}

Yasir Ali and Suleman Khan are shared first authors of this article.

\section{Conflicts of Interest}

The authors declare that they have no conflicts of interest.

\section{Authors' Contributions}

YA analyzed the data and participated in manuscript writing. YA, SK, NF, and AA collected blood samples and clinical data. YA, MA, and AA performed the laboratory experiments. FJ being a supervisor at AWKUM, Pakistan, helped in writing and editing the manuscript. YC, ZI, AAS, and $\mathrm{MJ}$ edited the manuscript. All authors read and approved the final manuscript.

\section{Acknowledgments}

The authors are highly thankful to the study subjects who participated in this study. The study was sponsored by Higher Education Commission, Pakistan, and School of Biomedical Sciences, The Chinese University of Hong Kong.

\section{References}

[1] D. Aletaha, "Rheumatoid arthritis classification criteria: an American College of Rheumatology/European League against Rheumatism collaborative initiative," Arthritis \& Rheumatism, vol. 62, no. 9, pp. 2569-2581, 2010.

[2] J. S. Smolen and D. Aletaha, "Rheumatoid arthritis therapy reappraisal: strategies, opportunities and challenges," Nature Reviews Rheumatology, vol. 11, no. 5, pp. 276-289, 2015.

[3] E. D. O. Roberson and A. M. Bowcock, "Psoriasis genetics: breaking the barrier," Trends in Genetics, vol. 26, no. 9, pp. 415-423, 2010.

[4] M. Traylor, "Genetic associations with radiological damage in rheumatoid arthritis: meta-analysis of seven genome-wide association studies of 2,775 cases," PLoS One, vol. 14 , no. 10 , 2019.

[5] H. Zhu, "Gene-based genome-wide association analysis in European and Asian populations identified novel genes for rheumatoid arthritis," PLoS One, vol. 11, no. 11, 2016.

[6] V. Goëb, P. Dieudé, O. Vittecoq et al., “Association between the TNFRII 196R allele and diagnosis of rheumatoid arthritis," Arthritis Research \& Therapy, vol. 7, no. 5, 62 pages, Article ID R1056, 2005.

[7] S. F. Jalil, A. Bhatti, F. Y. Demirci et al., "Replication of european rheumatoid arthritis loci in a Pakistani population," The Journal of Rheumatology, vol. 40, no. 4, pp. 401-407, 2013.

[8] M. Pierer, S. Kaltenhäuser, S. Arnold et al., "Association of PTPN22 1858 single-nucleotide polymorphism with rheumatoid arthritis in a German cohort: higher frequency of the risk allele in male compared to female patients," Arthritis Research \& Therapy, vol. 8, no. 3, p. R75, 2006.

[9] R. Plenge and J. D. Rioux, "Identifying susceptibility genes for immunological disorders: patterns, power, and proof," Immunological Reviews, vol. 210, no. 1, pp. 40-51, 2006.

[10] M. Hiwatari, T. Taki, T. Taketani et al., "Fusion of an AF4related gene, LAF4, to MLL in childhood acute lymphoblastic leukemia with $t(2 ; 11)$ (q11;q23)," Oncogene, vol. 22, no. 18, pp. 2851-2855, 2003.

[11] M. Melko, D. Douguet, M. Bensaid et al., "Functional characterization of the AFF (AF4/FMR2) family of RNA-binding proteins: insights into the molecular pathology of FRAXE intellectual disability," Human Molecular Genetics, vol. 20, no. 10, pp. 1873-1885, 2011.

[12] S. Viatte and A. Barton, "Genetics of rheumatoid arthritis susceptibility, severity, and treatment response," in Seminars in ImmunopathologySpringer, Berlin, Germany, 2017.

[13] A. Barton, S. Eyre, X. Ke et al., "Identification of AF4/FMR2 family, member 3 (AFF3) as a novel rheumatoid arthritis susceptibility locus and confirmation of two further panautoimmune susceptibility genes," Human Molecular Genetics, vol. 18, no. 13, pp. 2518-2522, 2009. 
[14] Z. Luo, C. Lin, E. Guest et al., "The super elongation complex family of RNA polymerase II elongation factors: gene target specificity and transcriptional output," Molecular and Cellular Biology, vol. 32, no. 13, pp. 2608-2617, 2012.

[15] G. Orozco and A. Barton, "Update on the genetic risk factors for rheumatoid arthritis," Expert Review of Clinical Immunology, vol. 6, no. 1, pp. 61-75, 2010.

[16] A. Hinks and S. Eyre, "Association of the AFF3 gene and IL2/ IL21 gene region with juvenile idiopathic arthritis," Genes \& Immunity, vol. 11, no. 2, pp. 194-198, 2010.

[17] P. Prasad, "Caucasian and Asian specific rheumatoid arthritis risk loci reveal limited replication and apparent allelic heterogeneity in north Indians," PLoS One, vol. 7, no. 2, 2012.

[18] E. A. Stahl, S. Raychaudhuri, E. F. Remmers et al., "Genomewide association study meta-analysis identifies seven new rheumatoid arthritis risk loci," Nature Genetics, vol. 42, no. 6 , pp. 508-14, 2010.

[19] J. Freudenberg, H.-S. Lee, B.-G. Han et al., "Genome-wide association study of rheumatoid arthritis in Koreans: population-specific loci as well as overlap with European susceptibility loci," Arthritis \& Rheumatism, vol. 63, no. 4, pp. 884-893, 2011.

[20] D. Plant, E. Flynn, H. Mbarek et al., "Investigation of potential non-HLA rheumatoid arthritis susceptibility loci in a European cohort increases the evidence for nine markers," Annals of the Rheumatic Diseases, vol. 69, no. 8, pp. 1548-1553, 2010.

[21] J. Sambrook, E. F. Fritsch, and T. Maniatis, Molecular Cloning: A Laboratory Manual, Cold Spring Harbor Laboratory Press, Cold Spring Harbor, NY, USA, 1989.

[22] G. S. Firestein, "Evolving concepts of rheumatoid arthritis," Nature, vol. 423, no. 6937, pp. 356-361, 2003.

[23] I. B. McInnes and G. Schett, "The pathogenesis of rheumatoid arthritis," New England Journal of Medicine, vol. 365, no. 23, pp. 2205-2219, 2011.

[24] J. Karami, S. Aslani, A. Jamshidi, M. Garshasbi, and M. Mahmoudi, "Genetic implications in the pathogenesis of rheumatoid arthritis; an updated review," Gene, vol. 702, pp. 8-16, 2019.

[25] L. Klareskog, P. Stolt, K. Lundberg et al., "A new model for an etiology of rheumatoid arthritis: smoking may trigger HLADR (shared epitope)-restricted immune reactions to autoantigens modified by citrullination," Arthritis \& Rheumatism, vol. 54, no. 1, pp. 38-46, 2006.

[26] K. Kim, S.-Y. Bang, H.-S. Lee, and S.-C. Bae, "Update on the genetic architecture of rheumatoid arthritis," Nature Reviews Rheumatology, vol. 13, no. 1, pp. 13-24, 2017.

[27] Y. Kochi, Y. Okada, A. Suzuki et al., "A regulatory variant in CCR6 is associated with rheumatoid arthritis susceptibility," Nature Genetics, vol. 42, no. 6, pp. 515-519, 2010.

[28] F. S. Mohammadi, S. Aslani, S. Mostafaei, A. Jamshidi, P. Riahi, and M. Mahmoudi, "Are genetic variations in IL-21IL-23R-IL-17A cytokine axis involved in a pathogenic pathway of rheumatoid arthritis? Bayesian hierarchical metaanalysis," Journal of Cellular Physiology, vol. 234, no. 10, pp. 17159-17171, 2019.

[29] R. Arya, "Effects of covariates and interactions on a genomewide association analysis of rheumatoid arthritis," in Proceedings of the 4th International Conference on Global Health, Philadelphia, PA, USA, December 2009.

[30] M. N. Saad, M. S. Mabrouk, A. M. Eldeib, and O. G. Shaker, "Identification of rheumatoid arthritis biomarkers based on single nucleotide polymorphisms and haplotype blocks: a systematic review and meta-analysis," Journal of Advanced Research, vol. 7, no. 1, pp. 1-16, 2016.
[31] R. J. L. Tan, L. J. Gibbons, C. Potter et al., "Investigation of rheumatoid arthritis susceptibility genes identifies association of AFF3 and CD226 variants with response to anti-tumour necrosis factor treatment," Annals of the Rheumatic Diseases, vol. 69, no. 6, pp. 1029-1035, 2010.

[32] H. Cen, R.-X. Leng, W. Wang et al., "Association of AFF1 rs340630 and AFF3 rs10865035 polymorphisms with systemic lupus erythematosus in a Chinese population," Immunogenetics, vol. 64, no. 12, pp. 935-938, 2012.

[33] P. S. Ramos, "A comprehensive analysis of shared loci between systemic lupus erythematosus (SLE) and sixteen autoimmune diseases reveals limited genetic overlap," PLoS Genetics, vol. 7, no. 12, 2011.

[34] J. A. Todd and N. M. Walker, "Robust associations of four new chromosome regions from genome-wide analyses of type 1 diabetes," Nature Genetics, vol. 39, no. 7, pp. 857-864, 2007. 